\title{
Lärm und Herz-Kreislauf-Erkrankungen
}

\section{Noise and Cardiovascular Diseases}

\section{다 (2)(1) (-)}

Autoren

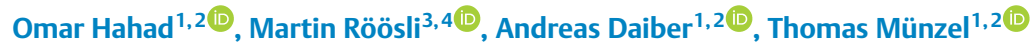

Institute

1 Zentrum für Kardiologie, Kardiologie I, Universitätsmedizin der Johannes Gutenberg-Universität Mainz, Mainz, Deutschland

2 Deutsches Zentrum für Herz-Kreislauf-Forschung (DZHK), Standort Rhein-Main, Mainz, Deutschland

3 Schweizerisches Tropen- und Public Health-Institut, Basel, Schweiz

4 Universität Basel, Basel, Schweiz

\section{Schlüsselwörter}

Lärm, Herz-Kreislauf-Erkrankungen, öffentliche Gesundheit

Key words

noise, cardiovascular disease, public health

Bibliografie

Aktuel Kardiol 2021; 10: 516-520

DOI 10.1055/a-1527-3145

ISSN 2193-5203

(C) 2021. The Author(s).

This is an open access article published by Thieme under the terms of the Creative Commons Attribution-NonDerivative-NonCommercial-License, permitting copying and reproduction so long as the original work is given appropriate credit. Contents may not be used for commercial purposes, or adapted, remixed, transformed or built upon. (https://creativecommons.org/licenses/by-nc-nd/4.0/).

Georg Thieme Verlag KG, Rüdigerstraße 14,

70469 Stuttgart, Germany

Korrespondenzadresse

Dr. Omar Hahad

Zentrum für Kardiologie, Kardiologie I

Universitätsmedizin der Johannes Gutenberg-Universität

Mainz

Langenbeckstraße 1

55131 Mainz, Deutschland

omar.hahad@unimedizin-mainz.de

\section{ZUSAMMENFASSUNG}

Umgebungslärm stellt einen bedeutsamen umweltbezogenen Risikofaktor für die öffentliche Gesundheit dar. Die Europäische Umweltagentur gibt an, dass mindestens 20\% der europäischen Bevölkerung schädlichen Tag-Abend-Nacht-Lärmpegeln von 55 Dezibel (dB) ausgesetzt sind, wobei die Weltgesundheitsorganisation zum Schutz der Bevölkerung, abhängig von der Lärmquelle, bis zu $10 \mathrm{~dB}$ tiefere Grenzwerte empfiehlt. Chronischer Lärm kann mit alltäglichen Aktivitäten und dem Schlaf interferieren und mentale sowie physiologische Stressreaktionen auslösen, die langfristig das Risiko für HerzKreislauf-Erkrankungen erhöhen können. Daher sind zwingend präventive Maßnahmen an der Quelle inklusive lärmreduzierender bautechnischer Veränderungen notwendig, um die Einhaltung von Lärmgrenzwerten zu gewährleisten und die Bevölkerung vor den negativen gesundheitlichen Auswirkungen des Lärms zu schützen.

\section{ABSTRACT}

Environmental noise is a significant environmental risk factor for public health. The European Environment Agency states that at least $20 \%$ of the European population are exposed to harmful day-evening-night noise levels of 55 decibels (dB), whereas the World Health Organization recommends up to $10 \mathrm{~dB}$ lower limit values, depending on the noise source, for the protection of the population. Chronic noise can interfere with daily activities and sleep and trigger mental and physiological stress reactions that can increase the risk of cardiovascular disease in the long term. Therefore, preventive measures at the source including noise-reducing structural changes are essential to ensure compliance with noise limits and to protect the population from the negative health effects of noise. 


\section{WAS IST WICHTIG?}

Lärm als Umweltrisikofaktor ist eine bedeutsame Ursache für die Krankheitslast und vorzeitige Todesfälle in der Bevölkerung. Chronische Lärmexposition kann mentale und physiologische Stressreaktionen auslösen, die zur Ausbildung und Progression von klassischen Herz-Kreislauf-Risikofaktoren beitragen. Die zunehmende aktuelle Evidenz legt nahe, dass Verkehrslärmexposition das Risiko für verschiedene Stoffwechsel- und Herz-Kreislauf-Erkrankungen bzw. HerzKreislauf-bedingte Sterblichkeit erhöhen kann, vermittelt über lärmbedingte Entzündungsprozesse und oxidativen Stress. Präventive systemische Maßnahmen, wie die von der Weltgesundheitsorganisation eingeführten Grenzwerte für Umgebungslärm, sind erforderlich, um die Krankheitslast durch Lärm in der Bevölkerung zu reduzieren.

\section{Lärm als bedeutsamer umweltbezogener Risi- kofaktor für die Gesundheit der Bevölkerung}

Umgebungslärm stellt einen der bedeutsamsten umweltbezogenen Risikofaktoren für die öffentliche Gesundheit dar. Gemäß einem Bericht der Europäischen Umweltagentur (EEA) sind in der EU 113 Millionen Personen Tag-Abend-Nacht-Straßenlärmpegeln $\left(L_{\text {den }}\right)$ von 55 Dezibel $(d B)$ oder mehr ausgesetzt, was $20 \%$ der europäischen Bevölkerung entspricht. Weiter sind 22 Millionen Personen von schädlichem Eisenbahnlärm, 4 Millionen von schädlichem Fluglärm und weniger als 1 Million von schädlichem Industrielärm betroffen [1]. Damit verursachen diese 4 Lärmquellen starke Lärmbelästigung bei 22 Millionen Personen sowie starke Schlafstörungen bei 6,5 Millionen Personen. Dies resultiert laut EEA jährlich in 48000 ischämischen Herzerkrankungen sowie 12000 vorzeitigen Todesfällen. Zusätzlich treten bei 12400 Kindern lärmbedingte kognitive Defizite auf. Neben den lärmbedingten gesundheitlichen Konsequenzen sind auch die damit in Verbindung stehenden gesamtgesellschaftlichen Kosten von signifikantem Ausmaß. Die Europäische Kommission (EC) beziffert die jährlichen sozioökonomischen Kosten in der EU, die durch die Kombination von Lärm und Luftverschmutzung, die beiden Umweltfaktoren mit den größten gesundheitlichen Auswirkungen in Europa, entste- hen, auf etwa 1 Billion Euro [2], wobei Lärm einen größeren Einfluss auf Indikatoren der Lebensqualität ausübt und Luftverschmutzung eher die Sterblichkeit beeinflusst [3]. Dagegen sind die sozioökonomischen Kosten, die durch Alkoholkonsum und Rauchen entstehen, mit 50-120 Milliarden bzw. mit 544 Milliarden relativ gering.

\section{KURZGEFASST}

Lärm, insbesondere durch Transport und Verkehr, ist ein bedeutsamer Risikofaktor für die öffentliche Gesundheit und geht mit erheblichen sozioökonomischen Kosten einher.

\section{Leitlinien der Weltgesundheitsorganisation (WHO) für Umgebungslärm}

In den aktuellen Leitlinien der WHO für Umgebungslärm für die Europäische Region von 2018 wurden Grenzwerte für Umgebungslärm aus verschiedenen Quellen definiert [4]. Hierzu wurden verschiedene Studien aus Europa, aber auch aus Amerika, Asien und Australien betrachtet und die Stärke der Empfehlung mittels GRADE-Methodologie (Grading of Recommendations Assessment, Development and Evaluation) hinsichtlich der lärmbedingten Auswirkungen auf Herz und Kreislauf sowie Stoffwechsel, Belästigung/ Ärgerreaktion, Schlaf, kognitive Beeinträchtigung, Hörschäden und Tinnitus, Fehlgeburten, Lebensqualität, psychische Gesundheit und Wohlbefinden bewertet. - Tab. 1 zeigt einen Überblick über die Grenzwerte/Empfehlungen in Abhängigkeit von der Lärmquelle, bei deren Überschreitungen langfristig mit schädlichen gesundheitlichen Auswirkungen zu rechnen ist. Da vor allem der Nachtlärm mit negativen gesundheitlichen Konsequenzen aufgrund der Beeinträchtigung des Schlafes verbunden ist, werden in den WHO-Nachtlärmrichtlinien für die Europäische Region im Allgemeinen Nachtlärmaußenpegel von weniger als $40 \mathrm{~dB}$ Lnight (Nachtlärmindex) gefordert [5]. Auch das Umweltbundesamt empfiehlt übergreifend Lärmpegel innerhalb von Wohnungen, die nachts unter $25 \mathrm{~dB}(\mathrm{~A})$ und tags unter $35 \mathrm{~dB}(\mathrm{~A})$ liegen. Diese Lärminnenpegel können bei gekippten Fenstern erreicht werden, wenn die Außenlärmpegel nachts unter $40 \mathrm{~dB}(\mathrm{~A})$ und tags unter $50 \mathrm{~dB}$ (A) liegen [6].

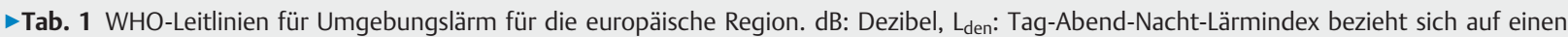
Zeitraum von 24 Stunden mit einem Zuschlag von 5 dB für den Abendzeitraum (19-23 bzw. 18-22 Uhr) und von $10 \mathrm{~dB}$ für den Nachtzeitraum (237 bzw. 22-6 Uhr), $L_{\text {night: }}$ Nachtlärmindex bezieht sich auf den Zeitraum von 23-7 bzw. 22-6 Uhr, $L_{\text {Aeq,24h: }}$ Lärmindex über einen Zeitraum von 24 Stunden. Die Grenzwerte beziehen sich auf die am stärksten lärmbelastete Außenfassade. Eine starke Empfehlung kann in den meisten Situationen als Politik angenommen werden, eine bedingte Empfehlung erfordert einen politischen Entscheidungsprozess mit substanzieller Diskussion und Einbeziehung verschiedener Akteure. Daten nach [4].

\begin{tabular}{|l|l|l|l|}
\hline Lärmquelle & Empfehlung $L_{\text {den }}$ & Empfehlung $\mathbf{L}_{\text {night }}$ & Stärke der Empfehlung \\
\hline Straßenverkehrslärm & $<53 \mathrm{~dB}$ & $<45 \mathrm{~dB}$ & stark \\
\hline Schienenverkehrslärm & $<54 \mathrm{~dB}$ & $<44 \mathrm{~dB}$ & stark \\
\hline Fluglärm & $<45 \mathrm{~dB}$ & $<40 \mathrm{~dB}$ & stark \\
\hline Windenergieanlagenlärm & $<45 \mathrm{~dB}$ & - & bedingt \\
\hline Freizeitlärm & $<70 \mathrm{~dB}\left(\mathrm{~L}_{\text {Aeq,24h }}\right)$ & - & bedingt \\
\hline
\end{tabular}




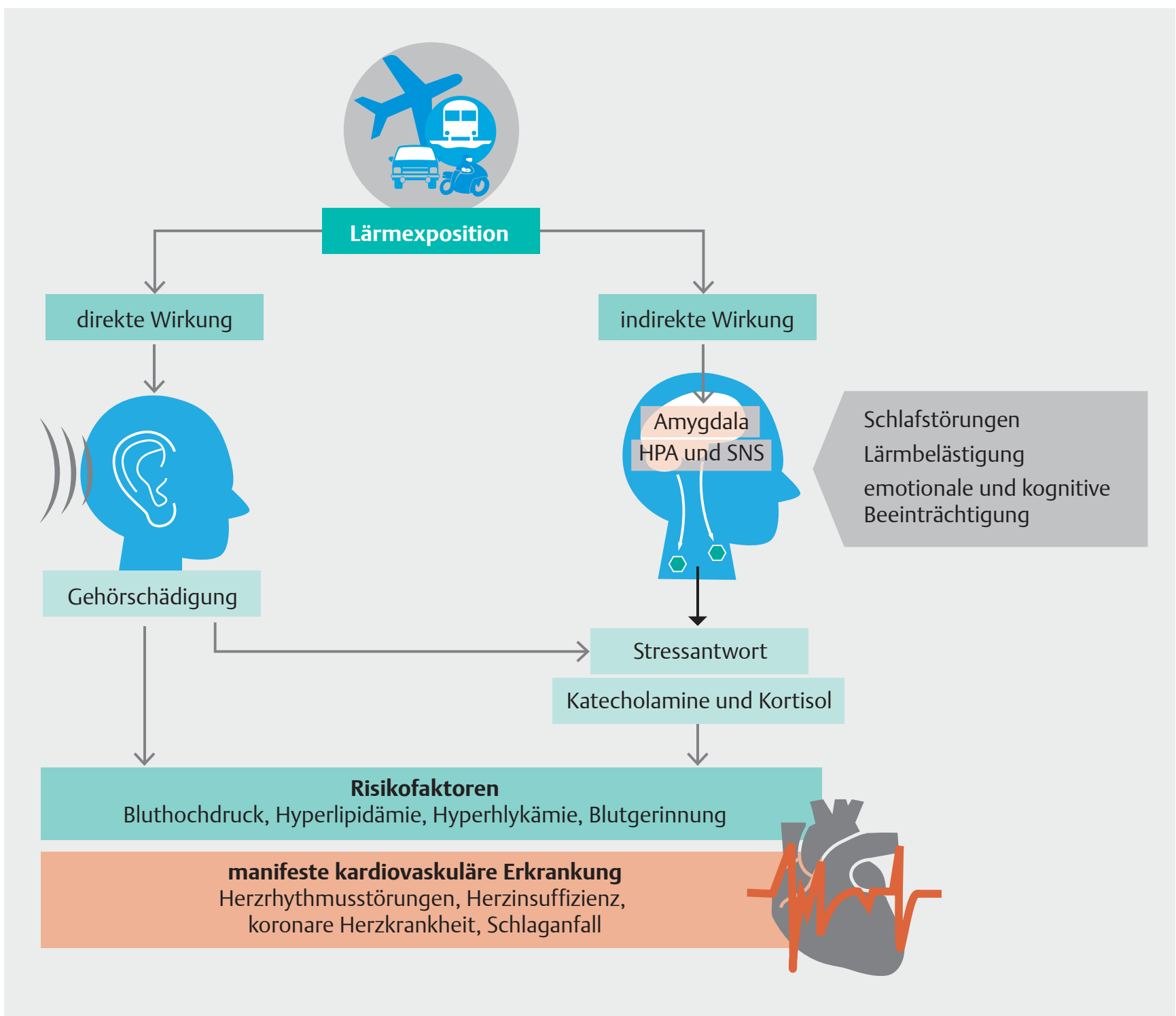

- Abb. 1 In dem Lärmwirkungsmodell von Babisch wird die indirekte Lärmwirkung als Ursache für die Ausbildung von Herz-Kreislauf-Risikofaktoren und -Erkrankungen beschrieben. Daten nach [8, 10]. HPA: Hypothalamus-Hypophysen-Nebennierenrinden-Achse, SNS: Sympathischen Nervensystem

\section{KURZGEFASST}

Um die Bevölkerung vor den negativen gesundheitlichen Konsequenzen von Lärm zu schützen, fordert die WHO die Einhaltung von quellenspezifischen und -unspezifischen Grenzwerten. Vor allem für den Nachtlärm, der als besonders gesundheitsschädlich gilt, werden übergreifende Lärmpegel von weniger als $40 \mathrm{~dB}$ gefordert, die jedoch bei einem erheblichen Teil der europäischen Bevölkerung überschritten werden.

\section{Lärm als psychosozialer Stressor}

Lärm wird allgemein als ein als störend empfundenes Geräusch verstanden, das als solches sowohl eine physikalische/objektive (Schallpegel) als auch eine psychologische/subjektive (Bewertung bzw. erlebte Belastung) Dimension besitzt. Das Stressmodell von Henry u. Stephens von 1977 [7] beschreibt die Verarbeitung von Stressoren in Abhängigkeit von der individuell wahrgenommenen Intensität sowie Bewertung der Stressoren über 2 verschiedene Gehirnareale und physiologische Stressachsen (Aktivierung der Amygdala und des sympathoadrenomedullären Systems bzw. des Hippocampus und des hypophysär-adrenokortikalen Systems). Darauf aufbauend beschreibt das Lärmwirkungsmodell von Babisch [8] die subjektive Wirkung von Lärm als psychosozialer Stressor als entscheidenden Faktor bei der Vermittlung von negativen gesundheitlichen Folgen ( $\triangleright$ Abb. 1). In diesem Sinne kann die indirekte (nicht auditorische) Lärmwirkung, die von der direkten (auditorischen) Lärmwirkung (z. B. Schädigung des Hörorgans durch sehr hohe Schalldruckpegel) unterschieden wird, über chronische Lärmexposition mit niedrigen Schallpegeln zu Störungen von Schlaf, Kommunikation und alltäglichen Aktivitäten führen und da- 
rüber kognitive und emotionale Stressreaktionen in Verbindung mit Lärmbelästigung auslösen. Eine anhaltende Lärmexposition und -belästigung führt zur Initiierung von physiologischen Stresskaskaden (vermittelt über einen erhöhten Stresshormonausstoß, Störung der zirkadianen Rhythmik, oxidativen Stress und Entzündungsreaktionen), die langfristig zu Gefäßschäden und kardiometabolischen Erkrankungen beitragen [9]. Basierend auf diesen Pathomechanismen erhöht Lärm das Risiko für Herz-Kreislauf-Erkrankungen wie Bluthochdruck, koronare Herzerkrankung, Herzinfarkt und Schlaganfall [10].

\section{KURZGEFASST}

Lärm als psychosozialer Stressor kann mentale und physiologische Stressreaktionen auslösen, die über längere Zeiträume zur Ausbildung und Progression von klassischen Herz-Kreislauf-Risikofaktoren beitragen können und darüber das Risiko für Herz-Kreislauf-Erkrankungen erhöhen können.

\section{Aktuelle Erkenntnisse aus der Lärmwirkungs- forschung zum Zusammenhang von Verkehrs- lärm und Herz-Kreislauf-Erkrankungen}

In einer Serie von tier- und humanexperimentellen Studien konnten wir zeigen, dass Verkehrslärmexposition mit einer Reihe pathophysiologischer Konsequenzen verbunden ist (für eine Übersicht siehe $[9,11])$. Zusammenfassend wurde in humanen Feldstudien beobachtet, dass simulierter Nachtfluglärm mit einer gestörten Gefäß- bzw. Endothelfunktion, einem erhöhten Stresshormonspiegel und einer deutlich verminderten Schlafqualität assoziiert war, wobei die tierexperimentellen Untersuchungen Entzündungsprozesse und oxidativen Stress durch eine vermehrte Bildung von reaktiven Sauerstoffspezies auf Zirkulations-, Gefäß-, aber auch auf zerebraler Ebene als Ursachen für die negativen Herz-KreislaufEffekte identifizieren konnten ( $\triangleright$ Abb. 2 ).

Auch in groß angelegten Beobachtungs- bzw. epidemiologischen Studien konnte der Einfluss von Verkehrslärm als Herz-Kreislauf-Risikofaktor nachgewiesen werden. In einer aktuelleren Metaanalyse aus dem Jahr 2018, die im Auftrag der WHO durchgeführt wurde, konnte ermittelt werden, dass Fluglärm das relative Risiko einer inzidenten koronaren Herzerkrankung um 9\% erhöht (95\%Konfidenzintervall $[\mathrm{KI}] 1,04 ; 1,15)$ pro Zunahme von $10 \mathrm{~dB} \mathrm{~L}_{\text {den }}$ [12]. In einer aktuellen Studie aus der Schweiz rund um den Flughafen Zürich war akuter Nachtfluglärm innerhalb von 2 Stunden mit einem erhöhten Risiko für einen Herz-Kreislauf-Tod (bestehend aus koronarer Herzerkrankung, Herzinfarkt, Herzinsuffizienz, Bluthochdruck, Schlaganfall und Herzrhythmusstörung) verbunden [13]. Dabei wurde ein 44\% höheres Risiko für einen Herz-KreislaufTod bei Personen beobachtet, die Fluglärmpegeln über $50 \mathrm{~dB}$ ausgesetzt waren (Odds Ratio 1,44; $95 \%-K I ~ 1,03 ; 2,04$ ) beim Vergleich von $>50 \mathrm{~dB}$ vs. $<20 \mathrm{~dB} \mathrm{~L}_{\text {Aeq }}$ (Lärmindex über einen Zeitraum von 24 Stunden). In einer weiteren Studie aus den USA wurde mittels Mediationsanalyse demonstriert, dass Verkehrslärm (Flug- und Straßenverkehrslärm) über eine erhöhte Aktivierung der Amygdala, die eine wichtige Rolle bei der emotionalen Verarbeitung von

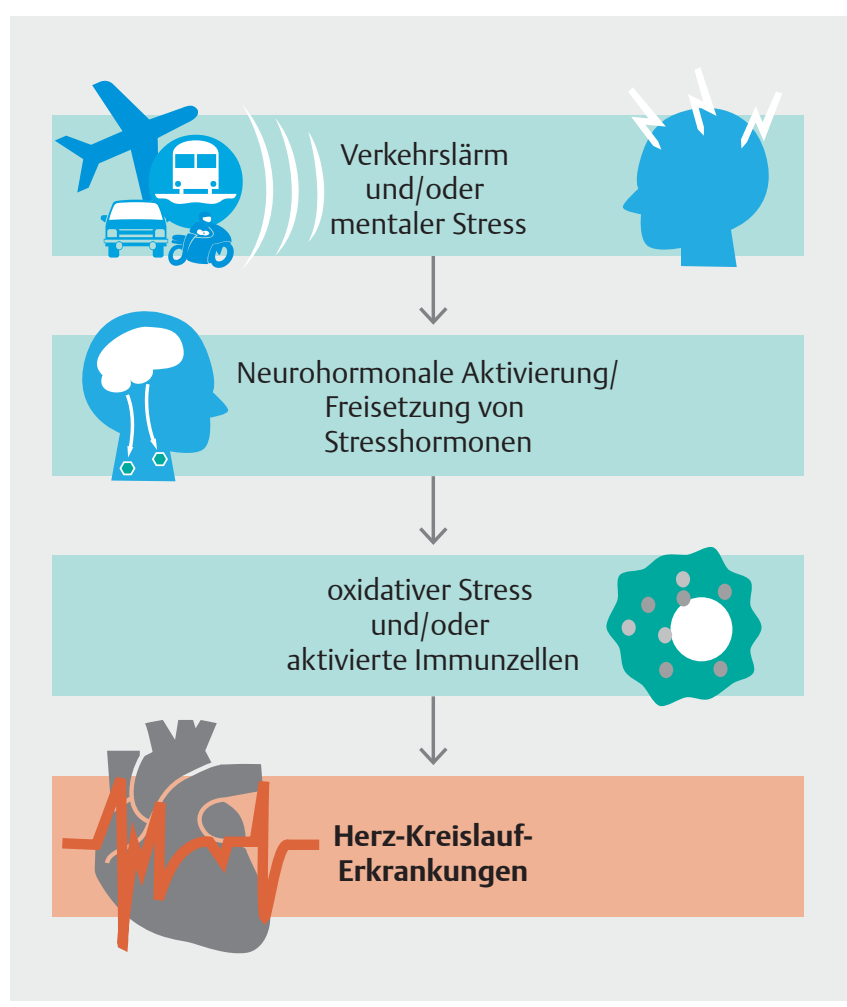

- Abb. 2 Lärm verursacht eine primäre Stressreaktion, die mit einer Freisetzung von Stresshormonen assoziiert ist. Diese Stressreaktion aktiviert entzündliche und/oder oxidative stressbasierte Signalwege und verursacht die Bildung von reaktiven Sauerstoffspezies und oxidativen Schäden, die wiederum die Ausbildung von Stoffwechselund Herz-Kreislauf-Risikofaktoren und -Erkrankungen begünstigen. Für eine Übersicht siehe [11].

Reizen spielt, mit einem erhöhten Risiko für Herz-Kreislauf-Ereignisse (bestehend aus Herz-Kreislauf-Tod, Herzinfarkt, instabile Angina pectoris, Schlaganfall, Herzinsuffizienz und koronare oder periphere Revaskularisation) einherging (relatives Risiko: 1,34; 95\%$\mathrm{KI}$ 1,15; 1,57 pro Zunahme von $5 \mathrm{~dB}[\mathrm{~A}] \mathrm{L}_{\text {Aeq,24h }}$ ) [14]. Zudem gibt es zunehmende experimentelle und epidemiologische Evidenz, dass Verkehrslärm den Metabolismus negativ beeinflusst und einen Risikofaktor für Diabetes und Übergewicht darstellt [15].

\section{KURZGEFASST}

Lärm initiiert eine Stressreaktion, die oxidativen Stress sowie Entzündungsreaktionen induziert und so zu Gefäßschäden und kardiometabolischen Erkrankungen (vor allem ischämischer Natur) beiträgt. Dabei spielt die Aktivierung der Amygdala eine wesentliche Rolle.

\section{Gesellschaftliche Konsequenzen}

Aufgrund der hinreichenden Evidenz aus aktuellen und früheren Studien sollte Umgebungslärm gesamtgesellschaftlich nicht nur als Gefahr für die öffentliche Gesundheit gesehen, sondern auch explizit als manifester Risikofaktor für Herz-Kreislauf-Erkrankungen 
anerkannt und entsprechend in den Leitlinien der medizinischen Fachverbände integriert werden. Im Gegensatz zu anderen klassischen Herz-Kreislauf-Risikofaktoren wie Rauchen, übermäßigem Alkoholkonsum oder körperlicher Inaktivität können umweltbedingte systemische Risikofaktoren wie Umgebungslärm kaum oder gar nicht durch das einzelne Individuum, Ärzte und Gesundheitsdienstleister beeinflusst werden. Diese Faktoren können nur durch systemische und politische Entscheidungen nachhaltig mittels Verhältnisprävention reduziert werden, die auf eine konsequente Einhaltung von Lärmgrenzwerten wie von der WHO empfohlen oder städte-/straßenbauliche Maßnahmen abzielen, um die Bevölkerung vor den negativen gesundheitlichen Auswirkungen des Lärms zu schützen.

\section{Fazit}

Lärm sollte als manifester Risikofaktor für Herz-Kreislauf-Erkrankungen anerkannt und entsprechend in den Leitlinien der medizinischen Fachverbände integriert werden. Präventive Maßnahmen wie die strikte Einhaltung von Lärmgrenzwerten oder städte-/ straßenbauliche Maßnahmen sind notwendig, um die Bevölkerung vor lärmbedingten Auswirkungen zu schützen.

\section{Interessenkonflikt}

Die Autorinnen/Autoren geben an, dass kein Interessenkonflikt besteht.

Literatur

[1] Europäische Umweltagentur. Lärm in Europa - 2020. 2020. Im Internet (Stand: 03.09.2021): https://www.eea.europa.eu/publications/environmental-noise-in-europe

[2] Europäische Kommission. Zusammenhänge zwischen Lärm und Luftverschmutzung und sozioökonomischem Status. 2016. Im Internet (Stand: 03.09.2021): https://ec.europa.eu/environment/integration/research/ newsalert/pdf/air_noise_pollution_socioeconomic_status_links_IR13_ en.pdf

[3] Vienneau D, Perez L, Schindler C et al. Years of life lost and morbidity cases attributable to transportation noise and air pollution: A compara- tive health risk assessment for Switzerland in 2010. Int J Hyg Environ Health 2015; 218: 514-521. doi:10.1016/j.ijheh.2015.05.003

[4] Weltgesundheitsorganisation. Leitlinien für Umgebungslärm für die Europäische Region. 2018. Im Internet (Stand: 03.09.2021): https://www. euro.who.int/_data/assets/pdf_file/0011/383924/noise-guidelinesexec-sum-ger.pdf

[5] Munzel T, Kroller-Schon S, Oelze M et al. Adverse Cardiovascular Effects of Traffic Noise with a Focus on Nighttime Noise and the New WHO Noise Guidelines. Annu Rev Public Health 2020; 41: 309-328. doi:10.1146/ann urev-publhealth-081519-062400

[6] Umweltbundesamt. Straßenverkehrslärm. 2020. Im Internet (Stand: 03.09.2021): https://www.umweltbundesamt.de/themen/verkehr-laerm/verkehrslaerm/strassenverkehrslaerm\#gerauschbelastung-im-strassenverkehr

[7] Henry JP, Stephens PM. Stress, Health and the social Environment: a sociobiological Approach to Medicine. Berlin: Springer; 1977.

[8] Babisch W. The Noise/Stress Concept, Risk Assessment and Research Needs. Noise Health 2002; 4: 1-11

[9] Munzel T, Sorensen M, Daiber A. Transportation noise pollution and cardiovascular disease. Nat Rev Cardiol 2021; 18: 619-636. doi:10.1038/s 41569-021-00532-5

[10] Hahad O, Kroller-Schon S, Daiber A et al. The Cardiovascular Effects of Noise. Dtsch Arztebl Int 2019; 116: 245-250. doi:10.3238/arztebl.2019. 0245

[11] Hahad O, Prochaska JH, Daiber A et al. Environmental Noise-Induced Effects on Stress Hormones, Oxidative Stress, and Vascular Dysfunction: Key Factors in the Relationship between Cerebrocardiovascular and Psychological Disorders. Oxid Med Cell Longev 2019; 2019: 4623109. doi:1 $0.1155 / 2019 / 4623109$

[12] Kempen EV, Casas M, Pershagen G et al. WHO Environmental Noise Guidelines for the European Region: A Systematic Review on Environmental Noise and Cardiovascular and Metabolic Effects: A Summary. Int J Environ Res Public Health 2018; 15: 379. doi:10.3390/ijerph15020379

[13] Saucy A, Schaffer B, Tangermann L et al. Does night-time aircraft noise trigger mortality? A case-crossover study on 24886 cardiovascular deaths. Eur Heart J 2021; 42: 835-843. doi:10.1093/eurheartj/ehaa957

[14] Osborne MT, Radfar A, Hassan MZO et al. A neurobiological mechanism linking transportation noise to cardiovascular disease in humans. Eur Heart J 2020; 41: 772-782. doi:10.1093/eurheartj/ehz820

[15] Munzel T, Sorensen M, Gori T et al. Environmental stressors and cardiometabolic disease: part I-epidemiologic evidence supporting a role for noise and air pollution and effects of mitigation strategies. Eur Heart J 2017; 38: 550-556. doi:10.1093/eurheartj/ehw269 